\title{
PARÁMETROS Y REGÍMENES DE HIDROTRANSPORTE DEL MINERAL LATERÍTICO APLICADOS EN LA EMPRESA MOA NÍQUEL S.A., HOLGUÍN, CUBA
}

(The Hydro Transport'S Rate and Parameters of the Lateritic Mineral Applied in the Moa Nickel S. A. Company, Holguín, Cuba)

\section{Raúl Izquierdo Pupo}

Instituto Superior Minero Metalúrgico de Moa, (ISMM), Holguín, Cuba, rizquierdo@ismm.edu.cu

(Recibido Agosto 20 de 2006 y aceptado Marzo 21 2007)

\section{Resumen:}

Revela los parámetros fundamentales de las instalaciones de hidrotransporte de pulpa cruda de la industria MoaNíquel, S.A. El trabajo contiene los resultados de la investigación sobre el hidrotransporte de la pulpa laterítica, realizada con el objetivo de obtener los parámetros que permitan diseñar y explotar las instalaciones de hidrotransporte del mineral laterítico fundamentadas técnica y económicamente.

La investigación experimental tuvo como escenario las instalaciones industriales en explotación y una instalación semiindustrial diseñada y construida en el ISMM. El comportamiento de los datos experimentales demostró la existencia de tres regímenes de movimiento de la pulpa laterítica: estructural, transitorio y turbulento.

Palabras clave: Hidrotransporte, Tuberías industriales.
Abstract

Here are exposed the installations' Fundamental parameters of the crude pulp hydro transit in the Moa NÍQuel S.A., Plant.

This paper contains the results of an investigation over the lateritic pulp hydro transit, that was realized in order to design and to exploit the installations of the hydro transit lateritic minerals, with a sound technique and economics.

The experimental research had as stage the industrial installations in exploitation and one semi-industrial installation, designed and built in I.S.M.M. According the experimental information the lateritic pulp has three types of movements: Structural, transitory and turbulent.

Key words: Hydro Transit, Industrial Pipelines.

\section{INTRODUCCIÓN}

D e las industrias del níquel, la empresa Moa-Níquel, S.A, es la única que aplica el hidrotransporte del mineral laterítico. El mineral procedente de la mina alimenta la planta de preparación de pulpa; donde se obtiene por un proceso de lavado y clasificación una pulpa con $25-30 \%$ de sólido en peso, la cual se hidrotransporta hasta los espesadores por una tubería de hormigón de $610 \mathrm{~mm}$ de diámetro y 5129 m de longitud.

La línea conductora está dividida en 54 partes, entre las cuales se encuentran pozos, donde la velocidad del flujo varía en los limites de 1-1,4 m/s. Experimentalmente se demostró que la velocidad de sedimentación de la mezcla en los espesadores, o sea, después de la hidrotransportación es inferior a la que posee antes del hidrotransporte. En el proceso de sedimentación se obtienen concentraciones de sólidos en la pulpa que varían generalmente entre $43 \%$ y $46 \%$ de sólido en peso.

La pulpa espesada se hidrotransporta, con la ayuda de bombas centrífugas hasta la planta de lixiviación, la tubería tiene una longitud de $460 \mathrm{~m}$ y un diámetro de $508 \mathrm{~mm}$, la velocidad media de movimiento de la pulpa varía entre los límites 0.3-0.6 $\mathrm{m} / \mathrm{s}$. Junto con el mineral laterítico se hidrotransporta una
Fracción gruesa de serpentina que representa un 5\% aproximadamente; parte de este material sedimenta en el fondo de la tubería, lo que provoca una disminución del diámetro de esta y un aumento de la resistencia hidráulica.

\section{MATERIALES Y MÉTODOS}

La explotación de estas instalaciones durante más de tres décadas permite deducir conclusiones de gran importancia para el diseño de nuevas instalaciones de hidrotransporte del mineral laterítico.

\subsection{Propiedades físico mecánicas del mineral y pulpa laterítica}

El mineral es una mezcla de lateritas de carácter terroso y serpentinas, parcialmente descompuesta y dura. El mineral laterítico se presenta como una tierra suelta y en terrones; y el mineral serpentinítico se presenta en toda la gama, desde el material terroso hasta fracciones de rocas duras de diversos tamaños.

En los yacimientos se distinguen cuatros zonas: la primera (3-8 $\mathrm{m}$ ) de potencia media, constituye las rocas de destape; en la segunda se encuentran las lateritas con una potencia media entre 5 y $15 \mathrm{~m}$, la tercera y cuarta están ocupadas por las 
serpentinas y rocas del basamento. El autor del trabajo fundamenta que las fases portadoras de níquel son goethita y espinelas y las no portadoras, hematitas y gibbsitas.

El níquel se concentra en la goethita en un contenido promedio de $1,4 \%$, concentrándose en la fracción $-0,045 \mathrm{~mm},(43 \%$ en peso) y de color amarillo parduzco. Las espinelas poseen un contenido promedio de $1,29 \%$ de $\mathrm{Ni}$, concentrándose en la fracción $(-0,83+0,074 \mathrm{~mm})$, representando el 5,6\% del material laterítico con color pardo oscuro. La gibbsita y las hematitas, fases no portadoras del $\mathrm{Ni}$ se concentran en las fracciones de $-83+0,074 \mathrm{~mm} \mathrm{y}-0,074+0,045 \mathrm{~mm}$ respectivamente.

El mineral empleado en la elaboración de este trabajo pertenece al yacimiento Atlantic, zona sur (L-2), y al frente de arranque (D-34), con la siguiente composición: $\mathrm{Ni}-1,20 \%$; Co $-0,06 \%$; $\mathrm{Fe}-44,18 \%$ y $\mathrm{Mg}-0,50 \%$.

El análisis granulométrico muestra que el $85 \%$ del mineral tiene un tamaño menor de $0,044 \mathrm{~mm}$, y el $15 \%$ restante está representado por impurezas, fundamentalmente, de granallas de hierro con un tamaño de 3-6,5 mm y pequeñas cantidades de serpentinas.

La determinación de la composición granulométrica de una muestra de mineral, y del diámetro medio de las partículas, se realizó por la siguiente metodología:

Determinación del peso de la muestra.

$$
\mathrm{Q}_{\text {min }}=0,02 \mathrm{~d}^{2}+0,5 \mathrm{~d} ;[\mathrm{Kg}]
$$

Se eligió una torre de tamices, conservando constante la razón (módulo) de las medidas de cada criba anterior a la siguiente, e igual a $\sqrt{2}$.

Después del cribado que se realiza por vía húmeda, se descanta cada muestra y se secan en una estufa. Cada clase constituye una muestra, la que se pesa y se determina el por ciento en peso respecto a la masa original.

El diámetro medio de la fracción de la muestra se determina por la fórmula siguiente:

$$
\mathrm{d}_{\mathrm{mi}}=\left(\mathrm{d}_{1}+\mathrm{d}_{2}\right) / 2
$$

donde: $\mathrm{d}_{1}$ : diámetro del mineral tamizado. $\mathrm{d}_{2}$ : diámetro del mineral retenido.

El diámetro medio para toda la muestra es:

$$
\mathrm{dm}=\left(\sum_{i=1}^{n} g i * d m i\right) / \mathrm{G}
$$

o

$$
\mathrm{dm}=\left(\sum_{i=1}^{n} \alpha i * d m i\right) / 100
$$

Donde: $\mathrm{g}_{\mathrm{i}}$ : peso de cada clase $\alpha i$ : contenido de la fracción, \% $\mathrm{d}_{\mathrm{mi}}$ : diámetro medio de la fracción $\mathrm{G}$ : peso total

Los resultados del análisis granulométrico realizado a una muestra de mineral con un peso total de $1013 \mathrm{Kg}$ aportan que el diámetro medio de las fracciones calculadas fue de $0,44 \mathrm{~mm}$.

Para la determinación de la densidad del sólido se aplicó la metodología del picnómetro, registrándose experimentalmente para el mineral analizado una densidad media de $3334 \mathrm{Kg} / \mathrm{m}^{3}$.

Durante los ensayos para determinar la velocidad de caída libre (tamaño hidráulico) de las partículas se observó que la sedimentación de las partículas en un medio en reposo es suave y sin la presencia de torbellinos, lo que permite asegurar que la sedimentación de las partículas en condiciones de reposo se efectúa en un régimen laminar. El número de Reynolds calculado a partir del diámetro de las partículas fue de $R_{e}<<2$.

La investigación de la velocidad de caída contrariada se realizó en una probeta de $200 \mathrm{~cm}^{3}$ y un diámetro de $91 \mathrm{~mm}$. La pulpa preparada con $20,25,30,40,45$ y $50 \%$ de sólido en peso se añadió a la probeta.

Las curvas que representan la relación entre la altura de descenso del sólido en el agua $(\mathrm{H})$ y el tiempo en que se efectúa este proceso $(\mathrm{t})$ muestran que a partir de la concentración de $30 \%$ aparece una zona casi paralela al eje del tiempo, donde la sedimentación es casi nula en comparación con la etapa inicial de las curvas; el fenómeno observado se explica por la aparición de propiedades estructurales en la pulpa a partir de una concentración superior a $30 \%$.

\subsection{Comportamiento reológico de la pulpa laterítica}

En numerosas fuentes bibliográficas se define reología como la ciencia del flujo y la deformación de los materiales. La reología de las pulpas lateríticas es un tema muy discutido. Investigaciones realizadas en la Universidad de Santiago de Cuba, dirigidas por el Dr. C. Leonel Garcel P., en un viscosímetro capilar, muestran que la curva reológica de la pulpa laterítica se ajusta al modelo Bulkley - Herschel, o sea, se corresponde con un líquido plástico real.

$$
\tau=\tau_{0}+\mathrm{k} * \gamma^{n}
$$

donde:

$\tau$ - esfuerzo de resistencia

$\gamma$ - gradiente de velocidad

$\tau_{0}$ - esfuerzo cortante inicial

$\mathrm{K}$ - índice de consistencia

$\mathrm{N}$ - índice de flujo 
El modelo de Bulkley-Herschel tiene la ventaja de describir la curva reológica completamente. En el presente trabajo se obtuvieron los parámetros reológicos de las pulpas lateríticas en un viscosímetro rotatorio. Los experimentos fueron realizados con pulpa de $30,35,40$ y $45 \%$ de sólido; la granulometría del mineral empleado fue de $-0,833 \mathrm{~mm}$ y agua destilada.

Los resultados se muestran con la función $\tau=f(\gamma)$. El gradiente que se registró en el viscosímetro es pequeño; menor de 100 $\mathrm{seg}^{-1}$. La recta para un $30 \%$ de sólido en peso corresponde con el modelo de Newton y las restantes concentraciones poseen una dependencia que se describen mediante el modelo de Bingham.

$$
\begin{array}{ll}
\tau=0.134 \gamma & \text { para } 30 \% \\
\tau=0.58+0.152 \gamma & \text { para } 35 \% \\
\tau=2+0.170 \gamma & \text { para } 40 \% \\
\tau=5.57+0.194 \gamma & \text { para } 45 \%
\end{array}
$$

Las suspensiones estructurales con densidad $\rho_{m}, y$ esfuerzo constante inicial $\tau_{0}$, pueden mantener en estado de suspensión partículas con diámetros (d) y densidad $\rho_{t}$.

$$
\left(\pi d^{3} / 6^{*} g^{*}\left(\rho_{t}-\rho_{m}\right)^{*} k=\pi^{*} d^{2} * \tau_{0}\right)
$$

donde:

$\mathrm{K}$ : coeficiente empírico que tiene en cuenta las desviaciones en las formas de las partículas respecto a una forma esférica.

Despejando d, de la expresión anterior se obtiene:

$$
d=\frac{6 * \tau_{0}}{g * k *\left(\rho_{t}-\rho_{m}\right)}
$$

Las partículas con tamaño menor de $\underline{\mathrm{d}}$, pueden mantenerse en suspensión, y las de mayor tamaño precipitan afectando las características estructurales de la suspensión.

\subsection{Estructura del flujo de una hidromezcla con una elevada concentración de sólido}

Como es conocido, el movimiento del agua y otros líquidos más viscosos como son los aceites lubricantes a bajas velocidades, transcurre en régimen laminar, el cual se subordina a la ley de Newton.

$$
\tau=-\mu *(\mathrm{dv} / \mathrm{dr})
$$

Donde: $\tau$ : esfuerzo de resistencia al movimiento. $\mu$ : coeficiente dinámico de viscosidad y $(\mathrm{dv} / \mathrm{dr})$ : gradiente de velocidad. La distribución de la velocidad en la sección del conducto se describe mediante una parábola.

$$
\mathrm{V}=\mathrm{V}_{\max }\left[1-(\mathrm{r} / \mathrm{R})^{2}\right]
$$

donde:

$R$ : radio del tubo

r: distancia medida a partir del eje de la tubería

$\mathrm{V}_{\max }: \operatorname{velocidad}$ máxima $(r=0)$

La complejidad en el movimiento de la hidromezcla, consiste en el complejo mecanismo de turbulencia, el que no es conocido hasta ahora, y también a un incompleto estudio de la suspensión de partículas en el líquido.

Para los líquidos homogéneos existe una serie de teorías que explican el régimen turbulento, una de ellas es la de PrandtlKarman. La ley de distribución de velocidad en la sección del flujo, según la teoría semiempírica de Prandtl-Karman.

$$
\left(\mathrm{V}_{\max }-\mathrm{V}\right) / \mathrm{V}_{*}=1 / \psi^{*} \ln (\mathrm{R} / \mathrm{y})
$$

$\psi$ : constante de Karman

y: distancia medida desde la superficie de la tubería

V: velocidad dinámica

Autores de diferentes trabajos han revelado que la presencia de grandes cantidades de partículas puede cambiar el régimen de flujo del líquido, cuando ellas se mueven con velocidades prácticamente iguales a la velocidad del líquido, disminuyendo la resistencia al movimiento. Durante el desplazamiento de la hidromezcla por la tubería, las partículas sólidas se trituran y se multiplican, aumentando la cantidad de pequeñas partículas suspensas en el medio.

La presencia de partículas sólidas en el flujo líquido varía sustancialmente el cuadro de la distribución de las velocidades en la sección transversal de la tubería. Es conocido que la distribución de la velocidad en la sección del flujo, cuando se mueve un líquido homogéneo en un tubo circular, horizontal, con una rugosidad uniforme, es simétrica para cualquier diámetro.

La distribución de la concentración de partículas sólidas por la altura del flujo se encuentra en una relación directa con la distribución de la velocidad en la sección del flujo. Por esto la distribución de la concentración del sólido en la sección del flujo de la hidromezcla tiene sus particularidades en función del tamaño hidráulico de las partículas, concentración de la hidromezcla y la velocidad media del flujo.

Cuando en el flujo se desplazan solamente pequeñas partículas y la concentración es pequeña, las partículas se distribuyen por la sección del conducto, presentándose una estructura similar a la de un flujo de líquido homogéneo, y el eje geométrico prácticamente coincide con el eje dinámico de la corriente.

Las hidromezclas con una concentración en masa superior a $25-30 \%$, formadas por partículas de pequeñas dimensiones adquieren propiedades particulares. En la mezcla comienzan a revelarse propiedades viscoso-plásticas, formándose una estructura sólida con el líquido. 
Las hidromezclas viscoso-plásticas, se describen generalmente por la ley de Shvedov-Bingham.

$$
\tau=\tau_{0}+\eta^{*}(\mathrm{dv} / \mathrm{dy})
$$

Donde:

$\eta$ : viscosidad de la hidromezcla.

\subsection{Modelo físico-matemático del flujo de la hidromezcla estructura}

Se ha demostrado de forma teórica y experimental la existencia en el flujo de agua de los regímenes: laminar, transitorio y turbulento. La corriente laminar es estrictamente ordenada, el líquido fluye por capas sin mezclarse, se describe por la ley de rozamiento de Newton y se determina completamente por esta.

Para poner en movimiento la pulpa estructural es necesario vencer un esfuerzo inicial $\tau_{0}$, por esta razón no se cumple la ley de rozamiento de Newton. Investigaciones realizadas en un viscosímetro capilar muestran que la curva reológica de la pulpa laterítica se comporta como un líquido plástico real.

De igual forma, se conoce que, cuando el gradiente de velocidad es mayor de $500 \mathrm{~s}^{-1}$ se establece una dependencia lineal entre el esfuerzo de resistencia $\tau$ y el gradiente de velocidad (dv/dr).

Los experimentos desarrollados en un viscosímetro rotatorio muestran que siendo el gradiente de velocidad menor de $100 \mathrm{~s}^{-1}$, también se establece una relación lineal entre el esfuerzo de resistencia y el gradiente de velocidad.

A partir de las condiciones anteriores se puede describir el flujo de la pulpa laterítica dividiendo la curva reológica en tres zonas:

1. Para esfuerzos $\tau_{0}\left\langle\tau\left\langle\tau_{a}\right.\right.$, el flujo se produce en régimen estructural, se conservan las propiedades estructurales de la pulpa.

2. En el intervalo de esfuerzos desde: $\tau_{a}$ hasta $\tau_{k}$, el flujo se efectúa en régimen transitorio, paulatinamente desaparecen las propiedades estructurales.

3. Para esfuerzos $\tau\rangle \tau_{k}$, el flujo se presenta en régimen turbulento.

Las tres zonas de la curva reológica se describen por el sistema de ecuaciones siguiente:

1. $\tau=\tau_{0}+\eta_{1} * \gamma ; \tau \in\left[\tau_{0}, \tau_{a}\right] ; \mathrm{n}=1$

2. $\tau=\tau_{0}+K * \gamma^{n} ; \tau \in\left[\tau_{a}, \tau_{k}\right]$

3. $\tau=\eta_{\text {min }} * \gamma \quad ;\left[\begin{array}{ll}\tau & \tau_{k}\end{array}\right] ; \mathrm{n}=1$

Investigaciones realizadas por Trainis dos décadas antes, sobre el traslado de hidromezclas de carbón por tubos confirmaron la existencia de los regímenes estructural, transitorio y turbulento. Se pueden apreciar los resultados experimentales en la determinación de la velocidad en diferentes puntos de la sección transversal de la tubería para velocidades medias del flujo 0,$5 ; 1,0$ y $2,0 \mathrm{~m} / \mathrm{s}$.

El modelo de Shvedov-Bingham, es el más aplicado para describir las hidromezclas plástico-viscosas.

$$
\tau=\tau_{0}+\eta^{*}(\mathrm{dv} / \mathrm{dy})
$$

Durante el movimiento uniforme de un líquido por un tubo se cumple:

$$
\tau=\frac{\Delta P}{2 L} * r
$$

donde:

$\Delta P=P_{1}-P_{2}$, caída de presión en la longitud $\mathrm{L}$.

Sustituyendo la ecuación (13) en (14) se obtiene:

$$
\frac{\Delta P}{2 L} * \mathrm{r}=\tau_{0}+\eta *(\mathrm{dv} / \mathrm{dr})
$$

donde:

$$
\mathrm{dv}=\frac{\Delta P}{2 L \eta} * \mathrm{r} * \mathrm{dr}+\frac{\tau_{0}}{\eta *} * \mathrm{dr}
$$

Integrando en los límites desde r hasta R obtendremos:

$$
\mathrm{V}=\frac{\Delta P}{4 L \eta} *\left(\mathrm{R}^{2}-\mathrm{r}^{2}\right)-\frac{\tau}{\eta}(\mathrm{R}-\mathrm{r})
$$

Cuando $\mathrm{r}=\mathrm{r}_{0}, \mathrm{v}=\mathrm{v}_{0}$ obtenemos:

$$
\mathrm{v}_{0}=\frac{\Delta P}{4 L \eta} *\left[\mathrm{R}^{2}-\left(\mathrm{r}_{0}\right)^{2}\right]-\frac{\tau}{\eta}\left(\mathrm{R}-\mathrm{r}_{0}\right)
$$

El flujo está compuesto de un núcleo con radio $\mathrm{r}_{0}$, con igual velocidad por toda su sección $\mathrm{v}_{0} \mathrm{y}$ una capa adyacente a la pared con una velocidad $\mathrm{v}$.

El gasto está formado por la suma de los gastos del núcleo $\mathrm{Q}_{0}\left(\mathrm{v}_{0}\right)$, más $\mathrm{Q}_{1}(\mathrm{v})$ para $\mathrm{r}>\mathrm{r}_{0}$.

$$
\begin{aligned}
& \mathrm{Q}=\mathrm{Q}_{0}+\mathrm{Q}_{1} \\
& \mathrm{Q}=\pi\left(\mathrm{r}_{0}\right)^{2} \mathrm{v}_{0}+\int_{r_{0}}^{R} 2 \pi \mathrm{rvdr}
\end{aligned}
$$

Sustituyendo las ecuaciones 17 y 18 en 19, e integrando, obtenemos la Ecuación de Bukingam.

$\mathrm{Q}=\frac{\pi * R^{4} * \Delta P}{8 * \eta * L} *\left[1-\frac{4}{3}\left(\frac{\tau_{0} * 2 L}{\Delta P * R}\right)+\frac{1}{3}\left(\frac{\tau_{0} * 2 L}{\Delta P * R}\right)\right]$

La Ecuación de Bukingam es difícil de resolver con relación a $\Delta P$, por eso de esta forma se utiliza muy poco para los cálculos ingenieriles. Si se desprecia el tercer sumando no se provoca un error sustancial.

$\mathrm{Q}=\frac{\pi * R^{4} * \Delta P}{8 * \eta * L} *\left[1-\frac{4}{3} *\left(\frac{\tau_{0} * 2 L}{\Delta P * R}\right)\right]$ 
La elaboración de los datos experimentales sobre el hidrotransporte con mezclas de carbón, arcilla, turba y otros materiales en tuberías con diámetros industriales demostró que para la determinación de las pérdidas hidráulicas se cumple la fórmula de Darcy-Beisbaja.

$$
\mathrm{i}=\frac{\Delta P}{L}=\lambda\left[\left(\mathrm{v}^{2}\right) /(2 \mathrm{D})\right] \rho
$$

donde:

$\lambda$ - coeficiente de resistencia hidráulica.

De la Ecuación 22, después de algunas transformaciones, se obtiene la ecuación para la determinación del coeficiente de resistencia hidráulica.

$$
\begin{gathered}
\lambda=64 * \eta *\left\{1+\left[\left(\tau_{0} * \mathrm{D}\right) /(\mathrm{N} * \eta * \mathrm{v})\right]\right\} /(\mathrm{v} * \mathrm{D} * \rho) \\
\lambda=64 / \mathrm{R}_{\mathrm{e}}^{*}
\end{gathered}
$$

donde:

$\mathrm{R}_{\mathrm{e}}^{*}=\mathrm{R}_{\mathrm{e}} /\left\{1+\left[\left(\tau_{0}^{*} \mathrm{D}\right) /\left(\mathrm{N} * \eta^{*} \mathrm{~V}\right)\right]\right\}=\mathrm{R}_{\mathrm{e}} /[1+(\mathrm{I} / \mathrm{N})]$

$\mathrm{I}=\left[\left(\tau_{0}^{*} \mathrm{D}\right) /\left(\mathrm{v}^{*} \eta\right)\right]$ criterio de Iluchina

El criterio de semejanza de Iluchina tiene en cuenta la relación entre las fuerzas plásticas y viscosas.

La magnitud $\mathrm{N}$ es una constante que depende de la hidromezclas, que varía en el rango de 2 a 8 según reportan diferentes investigadores. B. B. Trainis obtuvo que $\mathrm{N}=8$ para una hidromezcla de carbón.

En el trabajo se realiza el análisis para la determinación del coeficiente de pérdidas hidráulicas por medio del criterio modificado de $\mathrm{R}_{\mathrm{e}}^{*}$.

\subsection{Metodología aplicada al proceso de hidrotransportación}

La investigación de los parámetros y regímenes de hidrotransportación del mineral se realizaron en una instalación de dimensiones semiindustriales construidas en el ISMM de Moa.

El esquema de la instalación consta de los tanques 1 y 2, para la calibración del tubo Venturi y de recepción de la pulpa hidrotransportada respectivamente, las bombas centrífugas 3 y 4, los puntos de toma de presión 5, el tubo Venturi 6, los tramos de tuberías 7, 8 y 9, para determinar las pérdidas hidráulicas, la tubería 10 y 11 , en ascenso y descenso, respectivamente, el drenaje del sistema por la válvula 12, las válvulas de regulación 13 y 14, las piezas para variar el ángulo de inclinación 15, y las ventanas del cristal 16 y 18 .

Las tuberías 7, 8 y 9 poseen los diámetros 50,100 y 150 mm y longitud 20, 14,5 y $10 \mathrm{~m}$, respectivamente.

Los puntos para medir la presión se encuentran alejados de los extremos de la tubería a distancia igual o mayor de $40 \mathrm{D}$, para evitar las influencias de las perturbaciones más cercanas (codos, ventanas de cristal, etc.). El punto de observación del flujo de la pulpa (ventana de cristal) se encuentra situado a 5,5 m del tubo de Venturi.
El tanque 1 tiene un volumen de $1,9 \mathrm{~m}^{3}$ y el tanque 2 , de $2.9 \mathrm{~m}^{3}$, la bomba 3 tiene una productividad de $160 \mathrm{~m}^{3} / \mathrm{h}$ y la bomba 4 , de $60 \mathrm{~m}^{3} / \mathrm{h}$. Para los experimentos la instalación trabaja en circuito semicerrado (succión, impulsión y tanque), la limpieza se logra con el trabajo de la instalación en circuito abierto (succión, impulsión y drenaje).

Para eliminar el aire en cada medición se tomaron diferentes medidas, una de ellas fue la ubicación de trampas mediante las cuales se expulsaba el aire de la tubería a la atmósfera y otras de carácter práctico.

La determinación de la caída de presión que se produce durante el flujo de la mezcla por el tubo Venturi y por los tramos de medición de las pérdidas hidráulicas por rozamiento se realizó con la ayuda de manómetros diferenciales en forma de $U$, cuyo líquido manométrico es agua o mercurio en dependencia de la magnitud de la caída de presión.

$$
\begin{aligned}
& \Delta P=\left(L_{1}-L_{2}\right) \quad \text { para } \mathrm{H}_{2} \mathrm{O} \\
& \Delta P=\left(L_{1}-L_{2}\right) * 12.6 \quad \text { para } \mathrm{Hg}
\end{aligned}
$$

Donde $L_{1}$ y $L_{2}$ son unidades que descienden o ascienden el líquido en la escala del manómetro.

La pendiente hidráulica se determina:

$$
i=\frac{\Delta P_{r o z}}{L}
$$

Donde: $\Delta P_{r o z}$ caída de presión por rozamiento y $L$ longitud de la tubería.

El gasto de la hidromezcla se determina por medio del tubo Venturi, aplicando la fórmula:

$$
\mathrm{Q}=\mathrm{K} * \sqrt{\frac{\Delta P}{\rho}}
$$

Siendo: $\triangle P$ caída de presión y la constante del tubo Venturi $\mathrm{K}=0,0065$.

La constante del tubo de Venturi $\mathrm{K}$, se obtuvo mediante su calibración. Además, se determinó la concentración volumétrica de la hidromezcla $\mathrm{C}$, y la concentración en masa $\mathrm{S}$, aplicando las fórmulas siguientes:

$$
\mathrm{e}=w t / w t+w o ; \quad s=M t / M t+M o
$$

donde:

Wt y Mt - volumen y masa del sólido Wo y Mo - volumen y masa del agua

La cantidad de datos para cada medición se determinó según la metodología estadística siguiente:

Valor medio del parámetro:

$$
\mathrm{Vm}=\sum \varpi \mathrm{i} / \mathrm{n}
$$


Desviación media cuadrática:

$$
\delta^{2}=\sum_{i=1}\left(\mathrm{~V}_{\mathrm{i}}-\mathrm{V}\right)^{2}
$$

Número de datos:

$$
\mathrm{N}=\mathrm{t}^{2} \alpha(\mathrm{n}) \delta^{2} / \mathrm{m}^{2}
$$

donde:

t $\alpha(n)$ - coeficiente de student

$\alpha=0,05, \mathrm{t}=2$

Error permisible $\mathrm{m}=0,05$

\section{RESULTADOS}

La elaboración de los datos experimentales permitió establecer que para un $95 \%$ de confiabilidad es necesario tomar tres datos para cada medición.

Las investigaciones de los parámetros de hidrotransporte de la pulpa laterítica se realizaron para concentraciones en masas de 25, 30, 35, 40 y $45 \%$ de sólido.

Los resultados fueron elaborados según la dependencia $\mathrm{i}=\mathrm{f}(\mathrm{v})$ para el flujo de pulpa en los tubos 100 y $150 \mathrm{~mm}$ de diámetros (ver tabla 1).

Queda demostrado que durante el hidrotransporte de la pulpa laterítica se presentan los regímenes de movimiento: estructural, transitorio y turbulento; de esta forma, se confirman los resultados de las investigaciones de las propiedades reológicas y del modelo físico-matemático.

Las hidromezclas con una concentración superior a 35\% de sólido pertenecen a los líquidos no newtonianos, la distribución lineal de las rectas y su desplazamiento del centro de coordenadas manifiestan la presencia de una pulpa viscosoplástica. Cuando la velocidad de la pulpa es menor de $1 \mathrm{~m} / \mathrm{s}$ se observa el régimen estructural.

El régimen transitorio se manifiesta en los datos correspondientes a la segunda pendiente de las rectas. Los regímenes estructural y transitorio se caracterizan por diferentes grados de destrucción o rompimiento de la estructura. Las características estructurales de la hidromezclas pueden mantenerse aun a altos valores del gradiente de velocidad.

Durante el régimen turbulento, como se observa de la comparación entre los datos experimentales del flujo de la hidromezclas, y el comportamiento de los resultados para el flujo de agua, las dependencias $\mathrm{i}=\mathrm{f}(\mathrm{v})$ tienen las mismas características y se diferencian por un incremento de las pérdidas hidráulicas por la presencia del mineral. En la tabla 1, se resumen los resultados de los experimentos.

En los ensayos se observó que las partículas del mineral laterítico se mantienen en suspensión para las velocidades y concentraciones de las mezclas estudiadas. Además las variaciones en las pendientes hidráulicas de las tuberías inclinadas y la
Tabla 1. Resultados de experimentos

\begin{tabular}{|c|c|l|}
\hline Diámetro del tubo, $\mathbf{m}$ & Velocidad, $\mathbf{m} / \mathbf{s}$ & Régimen \\
\hline 0,150 & $0-1.0$ & $\begin{array}{l}\text { Estructural S }>35 \% \\
\text { Turbulento S }<35 \%\end{array}$ \\
\hline 0.100 & \multirow{2}{*}{$0-2.12$} & $\begin{array}{l}\text { Estructural S }>35 \% \\
\mathrm{v}<1.0 \mathrm{~m} / \mathrm{s} \\
\text { Transitorio S }>35 \% \\
\text { V }>1.0 \mathrm{~m} / \mathrm{s} \\
\text { Turbulento } \mathrm{S}<35 \%\end{array}$ \\
\hline
\end{tabular}

horizontal $\left(i_{t}-i\right)$; en inclinaciones pequeñas, $\left(<10^{\circ}\right)$ son despreciables (ver tabla 2).

Generalización de los datos experimentales: las teorías modernas de hidrotransportación de partículas sólidas suspensas en un líquido no permiten la solución de tareas ingenieriles prácticas sin una investigación preliminar experimental.

En el estudio del movimiento de hidromezclas viscosoplásticas en tubos, muchos investigadores han planteado la existencia de diferentes grados de rompimiento de la estructura. A. E. Smoldriev, en su trabajo (1989), muestra que durante el movimiento de la mezcla agua-carbón, se presentan tres regímenes de movimientos: régimen estructural a velocidades pequeñas; con el aumento de la velocidad aparece el régimen transitorio, y la aparición del régimen turbulento está condicionado por varios factores, siendo el más importante el rompimiento de la estructura.

Diferentes autores, (Chichenko, 1951), durante el estudio experimental del flujo de materiales arcillosos y de construcción en diferentes diámetros apreciaron los regímenes estructural y turbulento. Ambos coinciden en que el régimen estructural se describe por el modelo de Shvedov-Bingham y se presenta a velocidades en el rango de 0 a $0.6 \mathrm{~m} / \mathrm{s}$.

A.E. Smoldriev muestra en qué medida la utilización del criterio generalizador de Reynolds $\left(\mathrm{R}_{\mathrm{e}}^{*}\right)$ es correcto para la determinación del coeficiente de pérdidas hidráulicas para diferentes regímenes de movimiento. Smoldriev, para la construcción de la dependencia gráfica $\lambda \underline{\text { vs }} \mathrm{R}_{\mathrm{e}}^{*}$, utilizó datos experimentales de diferentes autores y diferentes materiales. Los puntos experimentales para los regímenes estructural y transitorio se ajustan perfectamente a la línea $\lambda$ - $\left(64 / R_{e}^{*}\right)$, lo que confirma la racionalidad de utilizar el criterio de Reynolds para la generalización de los datos experimentales.

En el presente trabajo se analizan los regímenes estructural y turbulento tomando como base los parámetros y experiencias de las dos instalaciones que se encuentran en explotación por un período mayor de 30 años.

La preservación de la semejanza hidrodinámica es rigurosa, para ello los experimentos se realizaron con el mineral laterítico y con una granulometría $(-0.833 \mathrm{~mm})$. Las hidromezclas se prepararon con el agua que se emplea en el propio proceso y con las concentraciones de sólidos de explotación. 
Otro parámetro importante para conservar la semejanza que se tuvo en cuenta fue la velocidad del flujo. La variación del diámetro de la tubería con respecto a los diámetros industriales se realizó debido a los grandes diámetros que existen en la fábrica.

En el presente trabajo se emplean conocidos criterios de semejanzas. En el régimen estructural la presencia de una pulpa visco-plástica determinó que los criterios a emplear son los criterios de Reynolds e Iluchina.

$\mathrm{El}$ valor de $\mathrm{N}$ determinado experimentalmente para el mineral laterítico es de $\mathrm{N}=2.3$.

En el régimen turbulento la viscosidad tiene un significado secundario para la determinación de las pérdidas hidráulicas y el papel fundamental lo juega la fuerza de gravitación. En este régimen es conocido el criterio de Froude.

$$
F_{r}=\left(v^{2} / g \cdot D\right)
$$

La influencia de la fuerza gravitacional se puede obtener mediante el complejo adimensional, $\pi$.

$$
\pi=\frac{i}{\rho L g}
$$

o sea,

$$
\frac{i}{\rho_{m} g L}=\frac{i_{0}}{\rho_{0} g L},
$$

donde:

$$
i=i_{0} \frac{\rho_{s}}{\rho_{0}}=i_{0}(1+a c k)
$$

donde:

i - pérdidas específicas de energía hidráulica para la hidromezcla

$\mathrm{i}_{0}$ - pérdidas específicas para el flujo del agua

$\mathrm{k}$ - constante, $\mathrm{k}=3.312$, para la pulpa laterítica

$$
\begin{aligned}
& a=\frac{\rho_{s}-\rho_{0}}{\rho_{0}} \\
& c=\frac{\rho_{m}-\rho_{0}}{\rho_{0}}
\end{aligned}
$$

donde:

$a$ - densidad relativa del sólido

$c$ - concentración volumétrica

$\rho_{0}, \rho_{m}, \rho_{s}$ - densidad del agua, de la pulpa, y del sólido respectivamente

La investigación realizada aporta una metodología ingenieril para el cálculo de los parámetros de hidrotransporte de las hidromezclas con mineral laterítico; la aplicación de esta se completará a través de trabajos posteriores.
Tabla 2. Resultados experimentales.

\begin{tabular}{|c|c|c|c|c|c|c|}
\hline $\mathbf{N}$ & $\begin{array}{c}\text { Volumen } \\
\text { del tanque } \\
\mathbf{m}^{\mathbf{3}}\end{array}$ & $\begin{array}{c}\text { Volumen de } \\
\text { la tubería } \\
\mathbf{m}^{\mathbf{3}}\end{array}$ & $\begin{array}{c}\text { Masa del } \\
\text { mineral } \\
\mathbf{~ k g}\end{array}$ & $\begin{array}{c}\text { Concent. } \\
\text { en masa } \\
\mathbf{\%}\end{array}$ & $\begin{array}{c}\text { Concent. } \\
\text { en vol. } \\
\%\end{array}$ & $\begin{array}{c}\text { Densidad } \\
\mathbf{K g} / \mathbf{m}^{3}\end{array}$ \\
\hline 1 & 1.133 & 0.874 & 670 & 25 & 9.2 & 1215 \\
\hline 2 & 1.133 & 0.874 & 873 & 30 & 11.56 & 1270 \\
\hline 3 & 1.133 & 0.874 & 1085 & 35 & 14.22 & 1332 \\
\hline 4 & 1.133 & 0.874 & 1338 & 40 & 17.09 & 1399 \\
\hline 5 & 1.133 & 0.874 & 1673 & 45 & 20.30 & 1474 \\
\hline
\end{tabular}

\section{CONCLUSIONES}

En el presente se trabaja en el diseño de una metodología de cálculo de los parámetros de hidrotransporte fundamentada y confirmada experimentalmente. Los métodos de cálculos fundamentados en la ecuación de Shedov-Binghan exigen la introducción de coeficientes de corrección, basados en una investigación experimental.

Las investigaciones realizadas sobre las propiedades físicos mecánicas de la hidromezclas permitieron formular el modelo físico-matemático del flujo de la pulpa laterítica.

La investigación teórico-experimental del flujo de la pulpa laterítica permitió comprobar que dependiendo de la velocidad del flujo y la concentración del sólido se presentan los regímenes: estructural, transitorio y turbulento.

El cumplimiento de la investigación permitió:

Obtener la dependencia para determinar el coeficiente de resistencia hidráulica del movimiento del régimen estructural.

Obtener la expresión para calcular las pérdidas hidráulicas durante el movimiento de la hidromezclas en el régimen turbulento.

\section{REFERENCIAS}

Avotins P. V. y colaboradores (1979). Reología y manipulación de las pulpas lateríticas, Simposio Internacional. New York.

Bulina Y. (1960). Transporte de suspensiones estructurales de alta densidad por tubos, Dis. Dr. Ciencias Técnicas.

Chichenko R. (1951). Hidráulica de las suspensiones de arcilla, Baku.

Falcón H. J. (1983) Consideraciones sobre la sedimentación de la pulpa limonítica en la planta "Pedro Sotto Alba", Revista Minería y Geología.

Filatov B. V. (1954). Determinación de las propiedades reológicas de suspensiones de arcillas en condiciones de un flujo estable, Revista Coloide. T. 16, N. ${ }^{0} 2$. 
Garcell P. L. (1986) Comportamiento reológico de las pulpas lateríticas, III Encuentro Científico Técnico del Níquel.

Izquierdo P. R., Pérez B. R. y Nikolaev A. K. (1995). Investigación de los parámetros y regímenes racionales de hidrotransporte de las pulpas lateríticas aplicables a las condiciones de explotación de la Empresa Cmdte "Pedro Sotto Alba", Revista Minería y Geología.

Lautinov Z. F. y Filatov B. V. (1963). Aproximación de la ecuación de Bukingam al flujo de sistemas dispersos viscoso-plástica, Revista Coloide. T. XXV.

Pakroskaia B. N. (1985). Transporte hidráulico en la industria minera, Niedra.

Pérez B. R. (1971). Investigaciones experimentales de los parámetros del transporte hidráulico de minerales de hierro triturado, Revista Tecnología, N. ${ }^{\circ} 1$.
Pérez B. R. y Maliuk S. (1983). Algunos resultados de las investigaciones de los parámetros del transporte de sal común suspensa en flujos de salmueras saturadas, Revista Minería y Geología, N. ${ }^{\circ} 2$.

Rojas P. A. (1995). Principales fases minerales portadoras del níquel en los horizontes lateríticos del yacimiento Moa, Dis. Dr. Ciencias Técnicas.

Safonov Y. K. (1977). Modelos reológicos de suspensiones concentradas, Revista Trabajo San Petersburgo.

Skelland A. U. (1970). Non - Newtonian Flow And Heam Transfer, Edición Rev. La Habana.

Smoldriev A. E. (1980). Transporte hidráulico, Niedra.

Smoldriev A. E. y Safonov Y. K. (1989). Transporte por tuberías de hidromezclas concentradas, Moscú. Construcción de máquinas.

Trainis B. B. (1970). Parámetros y regímenes del transporte hidráulico de carbón por tubo. Nauka. 